\title{
beadplexr: Reproducible and automated analysis of multiplex bead assays
}

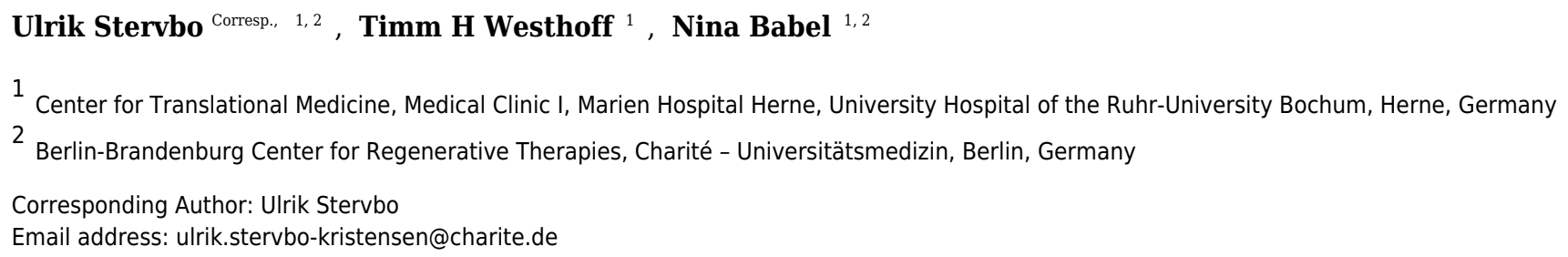

Multiplex bead assays are an extension of the commonly used sandwich ELISA. The advantage over ELISA is that they make simultaneous evaluation of several analytes possible. Several commercial assay systems, where the beads are acquired on a standard flow cytometer, exist. These assay systems come with their own software tool for analysis and evaluation of the concentration of the analyzed analytes. However, these tools are either tied to particular commercial software or impose other limitations to their licenses, such as the number of events which can be analyzed. In addition, all these solutions are 'point and click' which potentially obscures the steps taken in the analysis. Here we present beadplexer, an open-source R-package for the reproducible analysis of multiplex bead assay data. The package makes it possible to automatically identify bead clusters, and provides functionality to easily fit a standard curve and calculate the concentrations of the analyzed analytes. beadplexer is available from CRAN and from https://gitlab.com/ustervbo/beadplexr 


\section{1 beadplexr: Reproducible and automated 2 analysis of multiplex bead assays}

3 Ulrik Stervbo ${ }^{1,2}$, Timm H. Westhoff ${ }^{1}$, Nina Babel ${ }^{1,2}$

$4 \quad{ }^{1}$ Center for Translational Medicine, Medical Clinic I, Marien Hospital Herne, University

5 Hospital of the Ruhr-University Bochum, Herne, Germany

6 ²Berlin-Brandenburg Center for Regenerative Therapies, Charité - Universitätsmedizin,

7 Berlin, Germany

8 Correspondence

9 Ulrik Stervbo: ulrik.stervbo-kristensen@charite.de

\section{Abstract}

11 Multiplex bead assays are an extension of the commonly used sandwich ELISA. The

12 advantage over ELISA is that they make simultaneous evaluation of several analytes

13 possible. Several commercial assay systems, where the beads are acquired on a standard

14 flow cytometer, exist. These assay systems come with their own software tool for analysis

15 and evaluation of the concentration of the analyzed analytes. However, these tools are

16 either tied to particular commercial software or impose other limitations to their licenses,

17 such as the number of events which can be analyzed. In addition, all these solutions are

18 'point and click' which potentially obscures the steps taken in the analysis. Here we present

19 beadplexer, an open-source R-package for the reproducible analysis of multiplex bead assay

20 data. The package makes it possible to automatically identify bead clusters, and provides

21 functionality to easily fit a standard curve and calculate the concentrations of the analyzed

22 analytes. beadplexer is available from CRAN and from

23 https://gitlab.com/ustervbo/beadplexr. 


\section{Introduction}

25 The enzyme-linked immunosorbent assay (ELISA) is a commonly used method to

26 determine the concentration of soluble analytes such as cytokines (Elshal \& McCoy, 2006).

27 The concentration of the analyte is determined from a standard curve, which is created

28 from standard samples with known concentrations. The ELISA is a single point assay and

29 query into several analytes can be time consuming or impossible when the sample is

30 limited. Development in polystyrene bead preparations made it possible to construct

31 assays that allow for query of several analytes at the same time. Similar to the ELISA, the

32 analytes of interest are captured by a primary antibody (Figure 1A). The captured analytes

33 are subsequently labelled with a secondary antibody which in turn is detected with a

34 fluorochrome conjugated tertiary antibody. The level of fluorochrome intensity is directly

35 related to the amount of bound tertiary antibody, and therefore also to the amount of

36 analyte present in the sample. In a multiplex bead assay, the primary antibody is fixed on a

37 polystyrene bead, and physical properties such as size and granularity as well as

38 fluorescent colors of the beads are used to distinguish the different analytes studied. The

39 data is usually collected using a standard flow cytometer.

40 The LEGENDplex system from BioLegend, the CBA system from BD Biosciences, and the

41 MACSPlex system from Miltenyi Biotec are all bead based multiplex systems (Morgan et al.,

42 2004; Miltenyi Biotec, 2014; Yu et al., 2015). The systems differ slightly in terms of physical

43 properties and colors used, and in the number of analytes that can be simultaneously

44 identified. The Bio-Plex system from Bio-Rad works in a similar manner as those described

45 here, but requires a dedicated instrument and does not produce files suitable for analysis

46 with beadplexr. The individual assays that can be analyzed with beadplexr are described in

47 the following.

48

49

50

51

LEGENDplex: Beads fall into two large groups based on size and granularity - as related to the forward light scattering, FSC, and the perpendicular light scatter, SSC. Within each group, individual analytes are discriminated by the intensity of Allophycocyanin (APC) of the beads. The concentration of the analyte is related to the intensity of Phycoerythrin (PE). 
52 CBA: All beads have similar size and granularity. The individual analytes are discriminated

53 by the intensity of APC and APC-Cy7 of the bead. The concentration of the analyte is related 54 to the intensity of PE.

55 MACSPlex: All beads have similar size and granularity. The individual analytes are 56 discriminated by the intensity of PE and Fluorescein isothiocyanate (FITC) of the bead. The 57 concentration of the analyte is related to the intensity of APC.

58

All multiplex systems come with their own analysis software. However, these solutions might come with an added price tag because of binding to a particular piece of software, or the license is valid only for a number of bead events. In this case, large data files with many bead events or repeated re-evaluation of the acquired data might result an expiration of the license. In addition, the usability and flexibility of the analysis solutions are restricted and often impractical for experiments with a large number of samples. Currently no open source alternative exists.

Here the general usage of the beadplexr package for R (R Core Team, 2018) is introduced. It will be demonstrated how to load the files generated by the flow cytometer, identify bead populations, draw standard curves and calculate concentration of the experimental samples.

\section{Materials \& Methods}

The beadplexr package includes data from an unpublished "Human Growth Factor Panel (13-plex)" LEGENDplex (BioLegend) experiment performed in our laboratory. The dataset consists of eight controls samples and a serum sample from a single healthy volunteer. All samples were processed in duplicates and per manufacturer's instructions. The data was acquired on a CytoFLEX cytometer (Beckman Coulter). An example of a flow cytometry data file is also included in the package. We utilize these data to illustrate the functionality of the package.

The data here were analyzed with R, version 3.5.1, (R Core Team, 2018) and plots created with ggplot2 (Wickham, 2009) and cowplot (Wilke, 2017). The workflow and examples 
79 presented here make use of or suggests the following R-packages: devtools (Wickham,

80 Hester \& Chang, 2018), dplyr (Wickham et al., 2018), hexbin (Carr et al., 2018), magrittr

81 (Bache \& Wickham, 2014), purr (Henry \& Wickham, 2018), stringr (Wickham, 2018), and

82 tidyr (Wickham \& Henry, 2018).

\section{Results}

\section{Package overview}

85 The released package can be installed from CRAN and the development version from

86 GitLab:



98 The package provides several steps to extract the analyte concentration from the raw data 99 (Figure 1B). The functions for interacting with the data are flexible, but sensible defaults 100 make them accessible to the novice R-user. The workflow and examples presented here are 101 collected in Script S1, and a more detailed workflow is presented in the package vignette.

102 The latter can be viewed using the command vignette("legendplex-analysis").

\section{Reading FCS-filles}

104 beadplexr works with Flow Cytometry Standard (FCS) files (Seamer et al., 1997), which is 105 the usual output of a flow cytometer. The function read_fcs () loads the given FCS-file using 106 the functionality provided by the Bioconductor package flowcore (Ellis et al., 2017) and 107 performs the following steps: 
108

109

110

111

112

113

114

115

116

117

118

119

120

121

122

123

124

125

126

127

128

129

130

131

132

133

134

135

136

137

138

139

140

141

1. Apply an arcsinh transformation of the bead channels - this natural logarithm based transformation generally performs well on all flow cytometry data (Finak et al., 2010). Opposed to the traditionally used log10 scaling of flow cytometry data, the arcsinh can deal with the negative values produced by some newer digital flow cytometers

2. Remove boundary events of the size (FCS) and granularity (SSC) channels - events outside the range of the detectors are registered with the maximum value possible. These events can interfere with the clustering

3. Optionally subset the channels to contain just bead events - similar to removal of boundary events, this might improve identification of the bead clusters

4. Convert the FCS-data to a data. frame

\# Reading fcs-files

library(beadplexr)

\# Get the path to the example fcs-file

.file_name <- system.file("extdata",

"K2-CO7-A7.fCs",

package $=$ "beadplexr")

\#'read_fcs()' requires at least a path and file name of the file to load, \# by identifying the required forward and side scatter and the bead

\# property channels, only the required data is returned.

\#

\# The argument '.filter' takes a named list, where each element is a size

\# two vector, giving the lower and upper cut-off for the channel given in

\# the element name

. data <- read_fcs (

.file_name $=$.file_name,

.fSC_SSC $=c($ "FSC-A", "SSC-A"),

.bead_channels $=c($ "FL6-H", "FL2-H"),

.filter $=$ list (

"FSC-A" $=C(3.75 \mathrm{e} 5 \mathrm{~L}, 5.5 \mathrm{e} 5 \mathrm{~L})$,

"SSC-A" = $c(4 \mathrm{e} 5 \mathrm{~L}, 1 \mathrm{e} 6 \mathrm{~L})$,

"FL6-H" = $C(7 L$, Inf $)$ 
$142 \quad)$

143 )

144

145 Because of the variation in detector settings between flow cytometers, it is left to the user

146 to get the event filtering settings correct for an experiment. However, the event filtering

147 should remain stable once established. This, of course, requires that there is no change of

148 cytometer, and that there is no particular drift in the used cytometer. Visualizing the

149 populations greatly helps in setting the appropriate cut-offs (Figure 2). It is for this reason

150 that the ggplot2 based convenience function facs_plot() is included.

151 Naming the FCS-files

152 Each sample in a multiplex bead assay must have a unique and meaningful name. A later

153 step in the workflow separates standard samples from experimental samples. The standard

154 samples are in addition ordered in a way that calculation of dilution of standard

155 concentrations is possible. For the dataset included in the package, ' $\mathrm{C}$ ' followed by an

156 integer denotes the standard (control) samples - as suggested in the LEGENDplex manual -

157 and ' $\mathrm{S}$ ' followed by an integer denotes the experimental samples. The different parts of the

158 file name should be separated by a character not used in the IDs; this will make for easy

159 parsing of the file names.

\section{Identification of analyte MFI}

161 The mean fluorescence intensity (MFI) of each analyte relates directly to the concentration

162 of the analyte in the sample (Figure 1A). The first step to calculate the analyte

163 concentration is to identify the bead populations representing the analytes and calculate

164 the MFIs of these.

165 beadplexr makes use of structured Panel Information to provide analyte metadata such as

166 name and start concentration for each standard sample, as well as the name of the panel,

167 the fold dilution of the standards, and the units of the analytes. The desired Panel

168 Information is loaded using the load_panel() function by passing the name or a name

169 pattern to the function. The package itself comes with a set of LEGENDplex Panel

170 Information, which are documented in the help files to load_panel(). The Panel Information

171 file itself is in YAML format, and the load_panel() function can also load a Panel 
172 Information file located outside the package. The latter is useful in the cases of custom

173 panels. The Panel Information is not required, but makes sense if the assay is repeated 174 across several projects.

175

176

\# Libraries

177

178

library (beadplexr)

179 library(ggplot2)

180 library(cowplot)

181 library(dplyr)

182 library(purrr)

183 library(tidyr)

184 library(readr)

185 library(stringr)

186

187

188

\# Load data

189

190

data(lplex)

191

\# Load one of the panels distributed with the package, see ?load_panel() for

192 \# the included panels

193

panel_info <- load_panel(.panel_name = "Human Growth Factor Panel (13-plex)")

194

195 Analytes of any assay system are identified using the function identify_analyte(), which

196 identifies analyte clusters and assign an analyte ID to each cluster. The function takes a

197 data. frame with events and a character vector giving the name of column(s) where the

198 analytes can be discriminated. An identifier for each analyte is passed in the argument

199 .analyte_id, which is simply a character vector giving the ID of the analyte.

200 identify_analyte() sorts the clusters based on their centers and use this ranking to assign

201 the analyte IDs. The order of analyte IDs given in . analyte_id is therefore important and must

202 match the expected order of analytes. An optional argument is .trim which allows the

203 removal events in the periphery of a cluster. The value of the argument gives the fraction of 
204 the most distant points to be removed. Distance based trimming is non-trivial since the 205 possible numerical range depends on the detection range of the flow cytometer.

206 The function identify_analyte() interfaces several methods for unsupervised clustering, 207 which are passed in the .method argument. The default clustering method is clustering 208 large applications (clara) from the package cluster (Maechler et al., 2017). The method 209 selects a number of subsets of fixed size and applies the partitioning around medoids 210 (pam)-algorithm to each subset. The objective of the pam-algorithm is to minimize the 211 dissimilarity between the representative of $k$ clusters and the members of each cluster 212 (Kaufman \& Rousseeuw, 2009). The best resulting set of medoids (cluster centers) is that 213 with the lowest average dissimilarity of all points in the original dataset to the medoids.

214 Though similar to pam in algorithm type, the Base-R included kmeans works on minimizing 215 the distance to the cluster representative (Zaki \& Wagner Meira, 2014).

216 The dbscan method in the fpc package differs from clara and kmeans in that dbscan identifies 217 clusters based local density (Hennig, 2015). The function requires a neighborhood size and 218 minimum number of events in each neighborhood to evaluate whether points can be 219 considered as belonging to a cluster (Zaki \& Wagner Meira, 2014). If the bead populations 220 have different local densities, there is no guarantee that the correct number of clusters will 221 returned. This problem does not exist for Mclust from the mclust package, which fits a 222 Gaussian mixture model using the EM-algorithm (Scrucca et al., 2016). This algorithm 223 iteratively optimizes the individual parameters of $\mathrm{k}$ normal distributions (Zaki \& Wagner 224 Meira, 2014). This way the relationship between a cluster and a set of data points is given 225 by a set of probability scores.

226 We have found that dbscan() is the best clustering method for the forward-side scatter 227 population identification. However, it can be difficult to get the parameters event count and 228 neighborhood size correct. The reason for this difficulty lies in the sensitivity of the method 229 to the choice of neighborhood size; if it is too large clusters might be merged, and if it is too 230 small everything might be classified as noise. In our experience, the clustering function $231 \mathrm{clara}()$ is a great all-rounder although the subsampling performed by the function can lead 
232 to slight differences between each run. Using the same value for set.seed() at the

233 beginning of each session will alleviate this and make each run reproducible.

234 Different flow cytometers perform differently in terms of separation of the individual bead

235 populations. This is due to factors such as detector settings and age of the cytometer and its

236 light sources. The consequence is that the populations of interest might be closer together

237 or further apart. Another consequence might be an increased in the noise of the detectors

238 of the flow cytometer. Collectively these differences in the data constitution means that one

239 clustering function might perform better on one dataset while be inferior on another. As

240 with analysis of all flow cytometric data the optimal solution is a matter of taste, but the

241 better clustering function is the one that separates the populations well, without including

242 too much noise.

243 The function identify_legendplex_analyte() can be applied to each sample individually in a

244 loop. However, it is more prudent to apply the function to all samples at the same time

245 because the clustering decision will be identical for each sample. In addition, clustering on

246 all the samples is 1.4 times faster than clustering on each sample individually.

$254 \#$

255 \# The function requires a named list with analytes from the Panel

256 \# Information, and a list with a list of key-value pairs giving the arguments

257 \# for the bead identification on the forward and side scatter, and a list of

258 \# key-value pairs giving arguments for the bead identification in each

259 \# subpopulation in the APC channel.

$260 \#$

261 \# The argument .trim gives the fraction of events furthest from the centers of

262 \# the groups that should be removed. The population center is found by a

263 \# Gaussian kernel estimate. In this case we remove $1 \%$ and $3 \%$ of the of the 
264 \# events based on their distance to the group center.

$265 \#$

266 \# The inner lists can be named, but this is not required.

267 args_ident_analyte <- list(fs = list(.parameter = c("FSC-A", "SSC-A"),

\# The FCS-data is a list of samples, which we combine before cluster

275 \# identification.

276 analytes_identified <- 1plex \%>\%

277

278

279 bind_rows (.id = "Sample") \%>\%

280

The analyte IDs for the "Human Growth Factor Panel (13-plex)" bead group A are A4, A5, 282 A6, A7, A8, A10 and for group B the analyte IDs are B2, B3, B4, B5, B6, B7, B9. In this case, the beads are arranged from low to high, that is the lowest analyte ID has lowest intensity 284 in the APC channel (Figure 3).

285 This initial and crucial step of the analysis has been successfully performed with data from 286 a CBA experiment (C. McGuckin, CTIBIOTECH, Lyon, France, unpublished) and from a 287 MACSPlex experiment (Miltenyi Biotec, Bergisch Gladbach, Germany, unpublished) using 288 the function identify_analyte().

289 With the analytes identified and the bead populations documented, the MFI of each analyte 290 can finally be calculated. The function calc_analyte_mfi() gives the possibility to calculate 291 geometric, harmonic, and arithmetic mean of the in intensity of each respective analyte 292 reporter, such as PE in a LEGENDplex assay. Since the reporter intensities are usually log293 transformed only the geometric mean is relevant, but harmonic and arithmetic mean are 294 included to accommodate for special cases. 
296

297

\# The mean fluorescence intensity is calculated for each sample and analyte.

298 \# The function 'calc_analyte_mfi()' provides three ways of calculating the

299 \# MFI: geometric, harmonic, and arithmetic mean.

300 analyte_mfi <- analytes_identified \%>\%

301 filter(!is.na('Analyte ID`)) \%>\%

302 \# Call 'calc_analyte_mfi()' for each sample

303 group_by(Sample) \%>\%

304

305

306

307

308

309

310

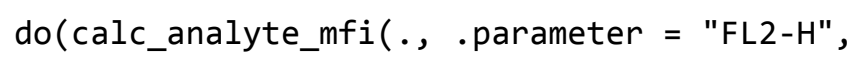

\# Later we will fit the standard curve on a log-log scale, so we transform \# here mutate $\left({ }^{\prime} F L 2-H^{`}=\log 10\left({ }^{`} F L 2-H^{`}\right)\right)$

311 The calculation of the concentration of the analytes of the experimental samples requires 312 two steps:

1. Create a standard curve by fitting a model to the MFI of the standard analytes and their known concentrations

2. Estimate the concentration of each sample analyte from the fitted model

316 The samples in the dataset included in the package can be distinguished by the presence of 317 ' $C$ ' or ' $S$ ', respectively. The sample type indicating letter is then followed by one or more 318 integers. Using this naming scheme, it is easy to separate standard samples from the 319 experimental samples. It is also easy to order the standard samples for concentration 320 assignment. In this case the naming scheme suggested in the LEGENDplex assay protocol is 321 followed: 7 indicates the highest concentration of the standard analyte, 1 indicates the 322 lowest concentration, and 0 indicates blank.

324 The order of the standard samples is crucial for the function calc_std_conc() to correctly 325 calculate the concentration of an analyte in each standard sample. The function requires a 
326 vector which gives the order of the standard samples, a start concentration for the analyte,

327 and a dilution factor. The standard samples are ordered numerically from high to low and

328 assigned a standard concentration, such that the first sample is given the start

329 concentration and the second to last sample the lowest concentration, and the very last

330 sample the concentration 0 , as this is assumed to be for background measurement.

331 The start concentration is stored in the Panel Information for each analyte separately, as

332 the start concentration might differ from analyte to analyte. The dilution factor is also given

333 in the Panel Information. It will always be the same for all standard analytes and is usually

3344 , meaning that the concentration of each standard analyte is 4 times lower than the

335 previous concentration. This generally gives a good range of standard concentrations.

336 \# Helper function to extract the sample number

338

\#' Cast sample ID to numeric

$339 \#^{\prime}$

340 \#' @param .s A string with the sample ID pattern to be cast

341 \#' @param .pattern A string giving the pattern

$342 \#$

343 \#' @return

344 \#' A numeric

$345 \quad \#^{\prime}$

346 as_numeric_sample_id <- function(.s, .pattern $=c(" c[0-9]+", " S[0-9]+"))\{$

347 .pattern <- match.arg(.pattern)

348

349

350

351

352

353

354

\# Extract the pattern defined just above, remove the first element, and

$355\}$

356

357

\# cast to a numeric

. $\%$ \% $\%$

358

359

\# We need to fit a standard curve on the standard samples, and use this curve 
360 \# to calculate the concentration of the experimental samples. Here we split

361 \# the data set in two: one with the standard samples and one with the

362 \# experimental samples.

$363 \#$

364 \# We need to order the standard samples from high to low in order to

365 \# calculate the concentration of the analytes in the standard sample.

366 \# Incorporating the information into the sample name in terms of an easily

367 \# parsable pattern is a good practice.

368

369 \# All standard samples have the pattern C[number]

370 standard_data <- analyte_mfi \%>\%

371 ungroup() \% \%

372 filter(str_detect(Sample, "C[0-9]+")) \% \%

373 mutate ('Sample number' = as_numeric_sample_id(Sample, . pattern = "C")) \% \%

374 select(-Sample)

375

376 \# All non-standards are experimental samples... we could also filter on

377 \# S[number]

378 experiment_data <- analyte_mfi \%>\%

379 ungroup() \% \%

380 filter(!str_detect(Sample, "C[0-9]+")) \%>\%

381 mutate ('Sample number' = as_numeric_sample_id(Sample, . pattern = "S")) \%>\%

382 select(-Sample)

383

384 \# To the standard data we have to add additional information such the start

385 \# concentration of each standard analyte and the dilution factor, as well as

$386 \#$ as the analyte names (analyte IDs by themselves do not make much sense).

$387 \#$

388 \# The concentration of the standard samples is calculated using

389 \#'calc_std_conc()', which take a vector of sample numbers for ordering, a

390 \# start concentration and a dilution factor.

391 standard_data <- standard_data \%>\%

392 left_join(as_data_frame_analyte(panel_info\$analytes), by = "Analyte ID") \%>\%

393 rename ('Analyte name` = name) \%>\%

394 group_by('Analyte ID`, 'Analyte name`) \%>\%

395 mutate(

396 Concentration = calc_std_conc ( 


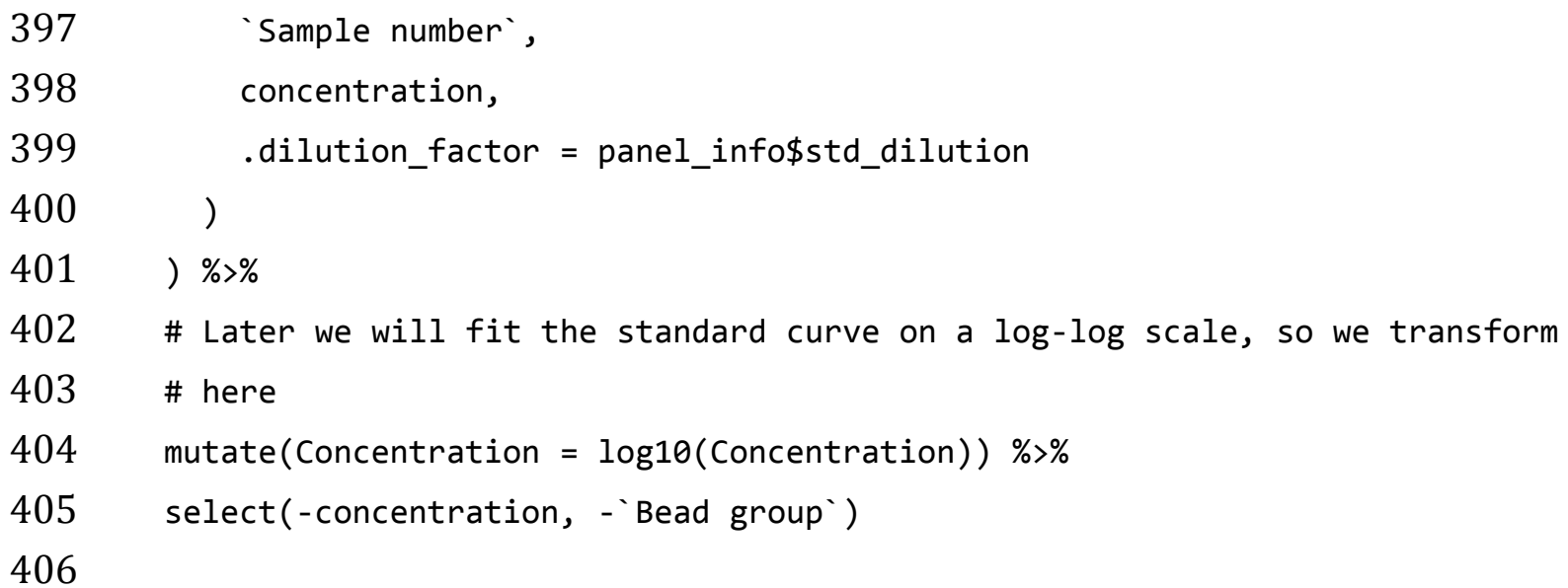

407 The next step is to fit a standard curve for each analyte. With the standard curve we can 408 calculate the concentration of the experimental samples (the purpose of the initial work), 409 we can check the quality of the measurements and the standard curve, and plot the 410 experimental samples on the standard curve (beadplexr provides easy access to all of this). 411 The latter is to allow for visual verification that the experimental samples are within the 412 linear part of the standard curve.

413 However, in each case we need to ensure that the correct standard curve is used with the 414 correct experimental data, which means we have to juggle at least three structures: A 415 data.frame with the standard data, a data. frame with the experimental sample data, and the 416 models for each analyte (probably a list). It quickly becomes tedious to ensure that 417 everything is in the correct order - and it is most certainly error prone. To circumvent this, 418 we can use the nest() and its inverse unnest() functions of the tidyr package. nest() relies 419 the fact that a data. frame in $\mathrm{R}$ is in fact a list, and uses this to pack a data. frame into a 420 single cell of a data. frame.

421 combine \# Nest standard and experimental data

423 \# Nested data.frames is a great way of combining and working with complex

424 \# data structures.

$425 \quad \#$

426 \# First we pack all the standard data in to a data.frame with a set of

427 \# data.frames

428 standard_data <- standard_data \%>\%

429 nest (-`Analyte ID`, .key = "Standard data") 
430

431

\# The the same for all the experimental data

432

433

experimental_data <- experiment_data \%>\%

434

435 nest (-`Analyte ID`, .key = "Experimental data")

436

\# Since both structures are data.frames we can easily combine them plex_data <- inner_join(standard_data, experiment_data, by = "Analyte ID")

437

438 With everything in a neatly arranged data. frame we can now focus on the actual task at 439 hand, namely calculation of the standard curve for each analyte. For this we use the 440 function fit_standard_curve(), which interfaces the drm() function from the drc package 441 (Ritz et al., 2015). The $\mathrm{drm}$ () function specializes in fitting various biological response442 models, and the drc package provides several response-models, such as the four- and five443 parameter log-logistic model. fit_standard_curve() is designed to be used in the piped 444 workflow, and takes a data.frame with MFIs and concentrations and returns the model as a 445 drc object. The four-parameter log-logistic model is widely used in analysis of ELISA data. 446 Since the five-parameter model yields better fits, because of the increased flexibility, this is 447 the default function (Gottschalk \& Dunn, 2005).

\# Calculate standard curves

\# For each of the analytes we calculate the standard curve. Working with

454 \# When clustering is performed with mclust, the package mclust is

455 \# loaded in the background (an unfortunate necessity). The mclust

456 \# package also has a function called 'map', so an unlucky side effect

457 \# of clustering with mclust, is that we need to be specify which map

458 \# function we use.

459

460 plex_data <- plex_data \%>\%

461

462

463

$$
\text { group_by(‘Analyte ID`) \%>\% }
$$$$
\text { mutate('Model fit' }=\text { purrr::map('Standard data', }
$$

fit_standard_curve, 
465

466 We can plot the standard curve using the built in plot_std_curve() function (Figure 4A).

467 With the standard curve created we can calculate the concentrations of the experimental 468 samples using the function calculate_concentration(), which requires a data.frame with 469 the MFIs in a column, and the fitted model. It can be helpful to apply 470 calculate_concentration() to the standard samples, as this can be used to verify that the 471 standard measurements were all fine, and that the estimation of the sample concentrations 472 therefore is trustworthy.

473 After calculating the concentrations we can plot the known standard concentrations versus 474 the estimated standard concentrations using the function plot_target_est_conc() (Figure $4754 \mathrm{~B}$ ) and visualize where the samples fall on the standard curve with plot_estimate()

476 (Figure 4C).

\# Calculate experimental sample concentrations

478

479

\# Using the standard curve just calculated, we can back-calculate the

484

485

purrr::map2('Standard data', 'Model fit', calculate_concentration, . parameter = "FL2-H")) \%>\%

486

487

488

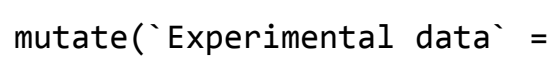

489

490 
497

498

499

500

501

502

503

504

505

506

507

508

509

510

511

512

513

514

515

516

517

518

519

520

521

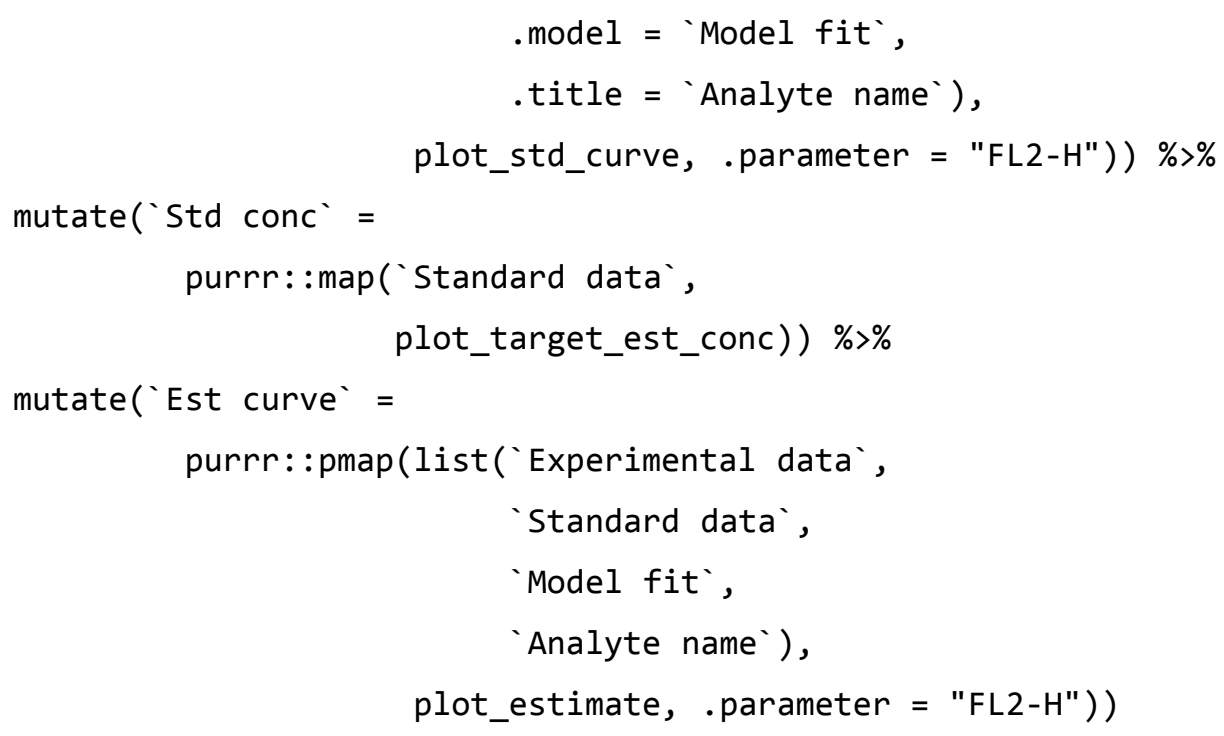

Lastly we fulfill the purpose of all the previous actions and extract the concentration of each analyte for each sample.

\# Extract analyte concentration

plex_data \%>\%

unnest('Experimental data`) \%>\%

\# Make the names a little more telling and transform them back to useful

\# concentrations

rename ('Concentration $(\mathrm{pg} / \mathrm{ml})^{`}=$ Calc.conc,

'Concentration error' = 'Calc.conc error') \%>\%

mutate ('Concentration $(\mathrm{pg} / \mathrm{ml})^{`}=10^{\wedge}$ 'Concentration $(\mathrm{pg} / \mathrm{ml})^{\prime}$ ',

'Concentration error' $=10^{\wedge}$ 'Concentration error')

\section{Discussion}

523 Multiplex bead assays make simultaneous evaluation of several analytes possible. Because

524 of this, they are an attractive alternative to the commonly used sandwich ELISA.

525 Commercial systems are available for acquisition on a standard flow cytometer, but these

526 commercial systems make use of their own proprietary software for the data analysis. This

527 can impose different limitations to the analysis. The R-package beadplexr, released under

528 the MIT license, is meant as an open-source alternative to these commercial systems. The

529 package is available from CRAN and from https://gitlab.com/ustervbo/beadplexr. 
530 A critical step in the analysis multiplex bead assays is the identification of bead populations

531 corresponding to each analyte. A single function in beadplexr acts as an interface to several

532 common, and tested, clustering functions, making it easy to find the best suited clustering

533 function. Future versions of the package will see improvements in this part, with inclusion

534 of other clustering methods and perhaps a heuristic for automatic method selection.

535 Flow cytometry data are inherently noisy. beadplexr only provides a rudimentary function

536 for removing points with no neighbors and lets the clustering functions determine which

537 events are considered noisy though the .trim argument. However, a very noisy data set

538 might make it difficult for an optimal identification of the bead clusters in the first place.

539 De-noising multidimensional data is not trivial, but work is planned in this direction for a

540 future release.

\section{Conclusion}

542 The R-package beadplexr provides a frame work for easy and reproducible analysis of

543 multiplex bead assays for the experienced and the novice user alike.

\section{Acknowledgments}

545 The authors wish to thank Miltenyi Biotec, Bergisch Gladbach, Germany and C. McGuckin,

546 CTIBIOTECH, Lyon, France for the example data to test the package. We further

547 acknowledge the support from the German Research Foundation (DFG) and the Open

548 Access Publication Fund of Charité - Universitätsmedizin Berlin.

\section{References}

550 Bache SM., Wickham H. 2014. magrittr: A Forward-Pipe Operator for $R$.

551 Carr D., Lewin-Koh ported by N., Maechler M., Sarkar contains copies of lattice functions

$552 \quad$ written by D. 2018. hexbin: Hexagonal Binning Routines.

553 Ellis B., Haaland P., Hahne F., Meur NL., Gopalakrishnan N., Spidlen J., Jiang M. 2017.

$554 \quad$ flowCore: flowCore: Basic structures for flow cytometry data. 
555 Elshal MF., McCoy JP. 2006. Multiplex Bead Array Assays: Performance Evaluation and 556 Comparison of Sensitivity to ELISA. Methods (San Diego, Calif.) 38:317-323. DOI: $557 \quad$ 10.1016/j.ymeth.2005.11.010.

558 Finak G., Perez J-M., Weng A., Gottardo R. 2010. Optimizing transformations for automated, 559 high throughput analysis of flow cytometry data. BMC Bioinformatics 11:546. DOI:

560 10.1186/1471-2105-11-546.

561 Gottschalk PG., Dunn JR. 2005. The five-parameter logistic: a characterization and 562 comparison with the four-parameter logistic. Analytical Biochemistry 343:54-65.

563 DOI: $10.1016 / \mathrm{j} . \mathrm{ab} .2005 .04 .035$.

564 Hennig C. 2015. fpc: Flexible Procedures for Clustering.

565 Henry L., Wickham H. 2018. purrr: Functional Programming Tools.

566 Kaufman L., Rousseeuw PJ. 2009. Finding Groups in Data: An Introduction to Cluster 567 Analysis. John Wiley \& Sons.

568 Maechler M., Rousseeuw P., Struyf A., Hubert M., Hornik K. 2017. cluster: Cluster Analysis $569 \quad$ Basics and Extensions.

570 Miltenyi Biotec 2014. Data acquisition and analysis without the MACSQuant@ Analyzer -$571 \quad$ General instructions for MACSPlex Cytokine Kits. Miltenyi Biotec.

572 Morgan E., Varro R., Sepulveda H., Ember JA., Apgar J., Wilson J., Lowe L., Chen R., Shivraj L., 573 Agadir A., Campos R., Ernst D., Gaur A. 2004. Cytometric bead array: a multiplexed 574 575 assay platform with applications in various areas of biology. Clinical Immunology 110:252-266. DOI: 10.1016/j.clim.2003.11.017.

576 R Core Team 2018. R: A Language and Environment for Statistical Computing. Vienna, 577 Austria. 
578 Ritz C., Baty F., Streibig JC., Gerhard D. 2015. Dose-Response Analysis Using R. PLOS ONE 10.

579 Scrucca L., Fop M., Murphy TB., Raftery AE. 2016. mclust 5: clustering, classification and 580 density estimation using Gaussian finite mixture models. The R Journal 8:205-233.

581 Seamer LC., Bagwell CB., Barden L., Redelman D., Salzman GC., Wood JCS., Murphy RF. 1997.

582 Proposed new data file standard for flow cytometry, version FCS 3.0. Cytometry

583

584 28:118-122. DOI: 10.1002/(SICI)1097-0320(19970601)28:2<118::AID-

585 Wickham H. 2009. ggplot2: elegant graphics for data analysis. Springer.

586 Wickham H. 2018. stringr: Simple, Consistent Wrappers for Common String Operations.

587 Wickham H., François R., Henry L., Müller K. 2018. dplyr: A Grammar of Data Manipulation.

588 Wickham H., Henry L. 2018. tidyr: Easily Tidy Data with "spread0" and "gather()" Functions.

589 Wickham H., Hester J., Chang W. 2018. devtools: Tools to Make Developing R Packages Easier. 590 Wilke CO. 2017. cowplot: Streamlined Plot Theme and Plot Annotations for "ggplot2."

591 Yu Y., Wang C., Clare S., Wang J., Lee S-C., Brandt C., Burke S., Lu L., He D., Jenkins NA., 592 Copeland NG., Dougan G., Liu P. 2015. The transcription factor Bcl11b is specifically 593 expressed in group 2 innate lymphoid cells and is essential for their development. $594 \quad$ The Journal of Experimental Medicine 212:865-874. DOI: 10.1084/jem.20142318.

595 Zaki MJ., Wagner Meira J. 2014. Data Mining and Analysis: Fundamental Concepts and $596 \quad$ Algorithms. Cambridge University Press. 
598 Figure legends

599 Figure 1: Overview of assay principle and the package workflow.

600 A) Schematic overview of the principle of a LEGENDplex assay. B) Steps in analysis of a

601 multiplex bead assay with accompanying visualizations.

602 Figure 2: Visualization of flow cytometry data.

603 Size (FSC) and granularity (SSC) can be used distinguish the two LEGENDplex bead

604 populations. A) Common monochrome scatter-plot created with facs_plot (.x = "FSC-H", $605 . y=$ "SSC-H", . beads = "Bead group") on the sample 'K3-C0-1.fcs'. High density regions

606 are obscured in this type of plots. B) Pseudo-colored scatter -plot created with

607 facs_hexbin (.x = "FSC-H", $. y=$ "SSC-H", . beads = "Bead group", .bins $=75)$ on the

608 same sample as in A). The number of events in discrete bins is indicated by color. The

609 coloring is according to the standard blue-green-yellow-red scheme, where blue indicates a

610 low number of events, and red indicates a high number. The Pseudo-colored scatter -plot

611 requires the R-package hexbin to be installed.

\section{Figure 3: Bead identification and visualization of LEGENDplex data.}

613 Populations identified in the sample 'K3-C0-1.fcs'. A) Identification of the two bead

614 populations ' $\mathrm{A}$ ' and ' $\mathrm{B}$ ' according to size and granularity: The two clusters were identified

615 using $\cdot$ method $=$ clara and noisy data points were excluded by $\cdot$ trim $=0.01$. B-C)

616 Identification of analytes of the bead population ' $A$ ' and ' $B$ ': The 1 dimensional clusters

617 along the APC channel were identified using $\cdot$ method = clara and noisy data points were

618 excluded by. trim $=0.03$. Noisy data points are assigned the group 'NA'.

619 Figure 4: Visualization of standard and experimental samples for Angiopoietin-2.

620 The dataset included in beadplexr is from a 13-plex assay. Here we use Angiopoietin-2 to

621 illustrate the visualizations. A) A log-log plot of the standard curve of Angiopoietin-2. Each

622 point is a single measurement (each in duplicate). The standard concentration is diluted in

623 steps of four fold dilution from $50,000.0$ to $12.21 \mathrm{pg} / \mathrm{ml}$. The intensity of the analyte is

624 measured in the PE channel. The full line indicates the best fit, and gray the confidence

625 interval. B) Correlation between the standard concentration (x-axis) and the calculated 
626 concentration of the standard samples (y-axis). The back calculation is done using the fit in 627 A) and the MFI of the samples. C) Using the fit in A) the concentration of an experimental 628 sample is calculated. Visual inspection of the position of the experimental samples on the 629 standard curve can reveal samples that are close to the upper or lower bound of the 630 standard curve.

631 
Figure 1 (on next page)

Overview of assay principle and the package workflow

A) Schematic overview of the principle of a LEGENDplex assay. B) Steps in analysis of a multiplex bead assay with accompanying visualizations. 

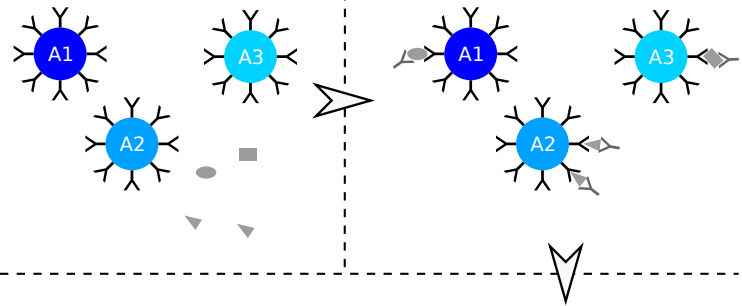

Incubate with tertiary antibody
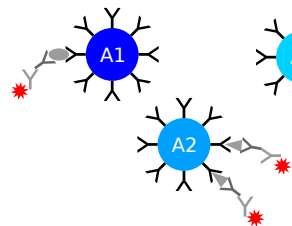

Incubate wiliesecondary antibody
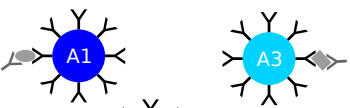

Load and process FACS-files

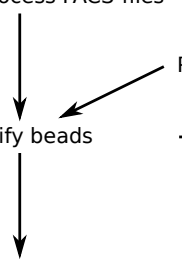

Fit standard curve

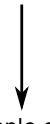

Calculate sample concentration

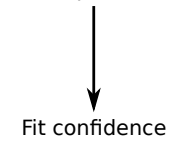

Fit confidence

\section{curve}

- Dot-plot

- Pseudo color plot

Parse panel information

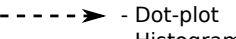

- Histogram

- Standard curve
- Expected vs. calculated
standard concentration - Analyte on standard curve 


\section{Figure 2 (on next page)}

\section{Visualization of FACS data}

Size (FSC) and granularity (SSC) can be used distinguish the two LEGENDplex bead populations. A) Common monochrome scatter-plot created with facs_plot(.x = "FSC-H", .y = "SSC-H", . beads = "Bead group") on the sample 'K3-C0-1.fcs'. High density regions are obscured in this type of plots. B) Pseudo-colored scatter -plot created with facs_hexbin(.x = "FSC-H", .y = "SSC-H", . beads = "Bead group", .bins = 75) on the same sample as in A). The number of events in discrete bins is indicated by color. The coloring is according to the standard blue-green-yellow-red scheme, where blue indicates a low number of events, and red indicates a high number. The Pseudo-colored scatter -plot requires the R-package hexbin to be installed. 
Figure 3 (on next page)

Bead identification and visualization of LEGENDplex data

Populations identified in the sample 'K3-C0-1.fcs'. A) Identification of the two bead populations ' $A$ ' and ' $B$ ' according to size and granularity: The two clusters were identified using $\cdot$ method $=$ clara and noisy data points were excluded by $\cdot$ trim $=0.01$. B-C) Identification of analytes of the bead population 'A' and ' $B$ ': The 1 dimensional clusters along the APC channel were identified using. method = clara and noisy data points were excluded by. trim $=0.03$. Noisy data points are assigned the group 'NA'. 


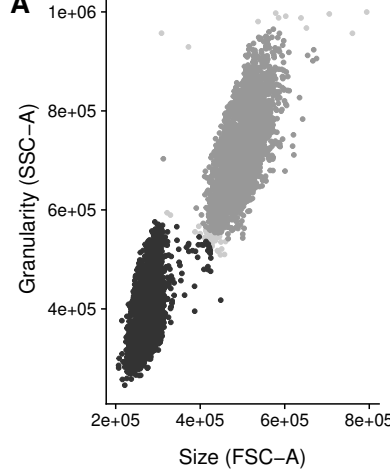

B PeerJ Group A
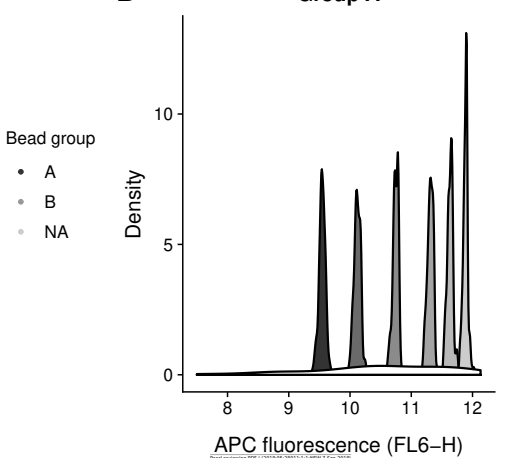

Group B
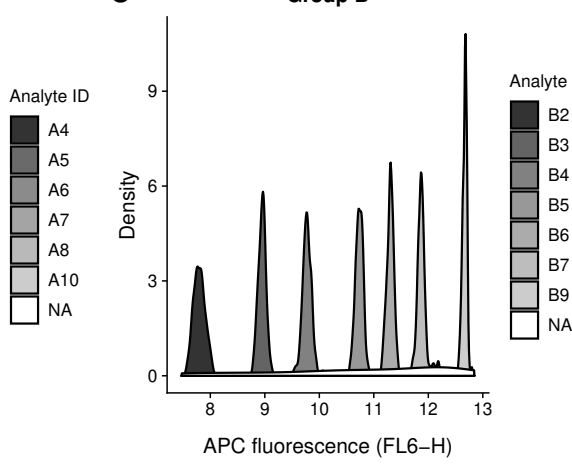


\section{Figure 4 (on next page)}

Visualization of standard and test samples for Angiopoietin-2

The dataset included in beadplexr is from a 13-plex assay. Here we use Angiopoietin-2 to illustrate the visualizations. A) A log-log plot of the standard curve of Angiopoietin-2. Each point is a single measurement (each in duplicate). The standard concentration is diluted in steps of four fold dilution from $50,000.0$ to $12.21 \mathrm{pg} / \mathrm{ml}$. The intensity of the analyte is measured in the PE channel. The full line indicates the best fit, and gray the confidence interval. B) Correlation between the standard concentration (x-axis) and the calculated concentration of the standard samples (y-axis). The back calculation is done using the fit in A) and the MFI of the samples. C) Using the fit in A) the concentration of an experimental sample is calculated. Visual inspection of the position of the experimental samples on the standard curve can reveal samples that are close to the upper or lower bound of the standard curve. 


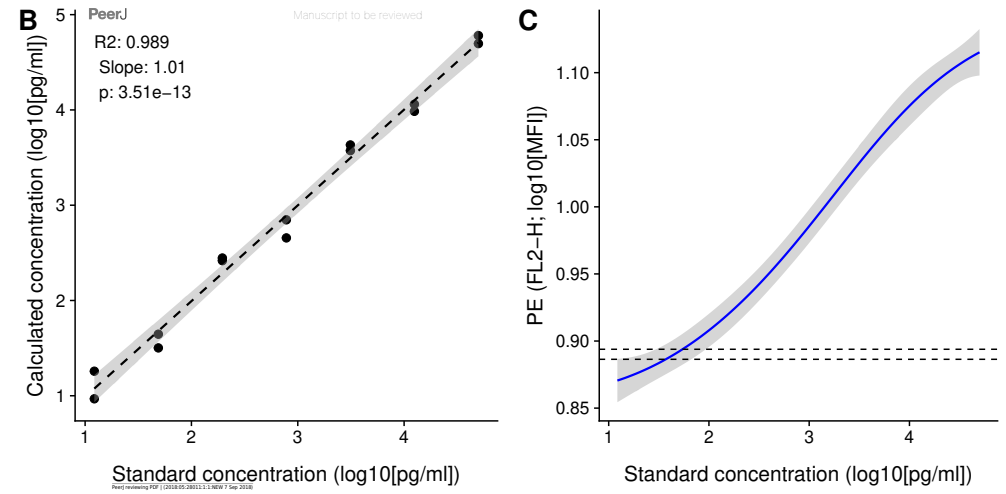

A

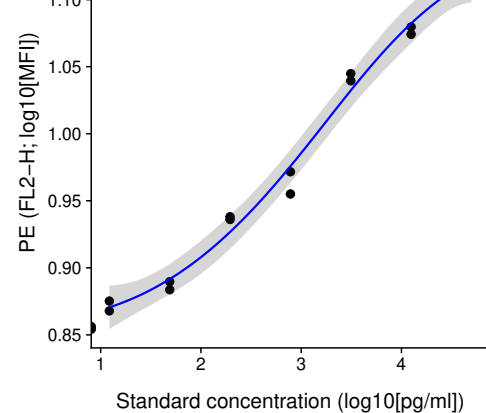

